\title{
The Vogt-Koyanagi-Harada syndrome: association with hypothyroidism and diabetes mellitus
}

\author{
Nattama Jaggarao, Douglas Voth* and Joergen Jacobsen
}

King Fahad Hospital, Al Baha, Saudi Arabia.

\begin{abstract}
Summary: A 45 year old Saudi male with poliosis, alopecia areata, vitiligo, anterior uveitis, inflammatory changes in the posterior pole of the retina and paraparesis presented with features of the Vogt-Koyanagi-Harada syndrome, uncontrolled diabetes mellitus and hypothyroidism. To the best of our knowledge the association of diabetes mellitus and hypothyroidism in this syndrome has not been reported previously.
\end{abstract}

\section{Introduction}

The Vogt-Koyanagi-Harada (VKH) syndrome is one of the uveomeningoencephalitic syndromes. It is associated with some systemic abnormalities and cutaneous manifestations of poliosis, vitiligo and alopecia. Jonathan Hutchinson in 1892 described a case of blanched eye lashes (poliosis), ${ }^{1}$ and 14 years later Vogt reported a similar case, ${ }^{2}$ Harada in 1926 described five cases of this syndrome including bilateral posterior uveitis and retinal detachment. ${ }^{3}$ Finally in 1929, Koyanagi described the interrelationships of headache, fever, bilateral uveitis, dysacusis, vitiligo, poliosis and alopecia. ${ }^{4}$ As far as we know the association of diabetes mellitus and hypothyroidism in the VKH syndrome has not been reported previously.

\section{Case report}

A 45 year old Saudi male was admitted with poorly controlled insulin dependent diabetes mellitus diagnosed 4 years earlier. One year prior to this admission after an episode of malaise, he developed poor vision, poliosis and vitiligo over a few days, according to the patient and it was confirmed by his brother. At about the same time he noticed that his scalp hair was falling out. A few weeks before admission he noticed that he had difficulty using his legs.

Correspondence: N.S.V. Jaggarao, M.R.C.P., Flat 18, 163 Cromwell Road, London SW5 0SQ, UK.

*Present address: Professor of Medicine, Oklahoma University Health \& Sciences Center, Associate Chief of Staff for Education, Oklahoma City Veterans Administration Medical Center, 921 Northeast 13th Street, Oklahoma City, OK 73104, USA.

Accepted: 1 December 1988
Examination revealed bilateral cataracts. There was anterior uveitis and the retinae showed inflammatory changes in the posterior pole. He had weakness and loss of sensation below T10 dermatome level. The deep reflexes in the lower limbs were decreased and the plantar reflex was equivocal. His voice was hoarse and there was non-pitting oedema but no goitre. He had alopecia areata of the scalp with vitiligo and white eye lashes. The thyroid stimulating hormone level was 105 $\mathrm{IU} / 1$ (normal 4). His total thyroxine was $33.5 \mathrm{nmol} / \mathrm{l}$ (normal $58-155 \mathrm{nmol} / \mathrm{l}$ ). The patient refused further neurological investigations, but had a cataract extraction. There was both clinical and biochemical response to thyroxine.

He was lost to follow-up for about 16 months but was readmitted again to improve the control of his diabetes mellitus which responded very well to the usual doses of insulin. Overall, his condition remained unchanged. Lumbar puncture was performed which yielded a clear, cell free cerebrospinal fluid with a raised protein content of $271 \mathrm{mg} / \mathrm{dl}$ (normal $15-45 \mathrm{mg}$ ). Thyroglobulin antibody was present in $1: 160$ dilution while the microsomal antibody titre was 1:1600. Parietal cell, smooth muscle and antimitochondrial antibodies were not detected.

\section{Discussion}

The VKH syndrome occurs in the third decade but cases have been reported at ages from 10 to $52^{5}$ in both sexes. There seems to be a statistically significant correlation between this syndrome and the HLA-BW $22 \mathrm{~J}$ haplotype in Japanese patients which is not found in other races. ${ }^{6}$ An association with ulcerative colitis, ${ }^{7}$ antibodies to myelin ${ }^{8}$ and ganglioside ${ }^{5}$ has also been reported.

(C) The Fellowship of Postgraduate Medicine, 1989 
In broad terms, there are three stages in the natural history of the VKH syndrome, namely, the meningeal, the ocular or ophthalmic, and the convalescent stages. The meningeal phase is characterized by combinations of headache, nuchal rigidity, seizures, hemi- or paraparesis, muscle weakness and psychosis. The cerebrospinal fluid acutely often shows lymphocytic pleocytosis. During the ocular phase which usually lasts for a few months but occasionally up to 10 years, there is uveitis and pain in the eyes; cataracts may also develop. Retinal detachment may develop following the accumulation of exudate in the sub-retinal space which may be absorbed later leading to retinal reattachment. Blindness can be a sequela which can possibly be prevented by corticosteroid therapy. In many patients, the convalescent phase is characterized by vitiligo, poliosis and alopecia with improvement in the uveitis. Alopecia is present in $60 \%$ of patients; sometimes generalized, it is usually limited to small areas. Poliosis is also seen in about the same proportion of the cases; it may appear suddenly a few weeks after the onset of the disease rather than in convalescence. Interestingly, vitiligo may occur within weeks after the onset of the condition, or even years beforehand.

This patient had poliosis, vitiligo, alopecia areata, anterior uveitis, inflammatory changes in the posterior pole of the retina and spinal cord disease suggesting the VKH syndrome.

Both autoimmunity ${ }^{9}$ and viral infections have been implicated in the aetiology of the VKH syndrome. Autoimmune mechanisms involving cytotoxic lymphocytes against melanocytes may be important as T-lymphocytes have been isolated from patients with

\section{References}

1. Hutchinson, J. A case of blanched eye lashes. Arch Surg 1892-1893, 4: 357.

2. Vogt, A. Fruhzeitiges Ergrauen Der Zilien Und Bemerkungen Uber Den Sogenannten Plotzlichen Eintritt Dieser Veranderung. Klin Monatsbl Augenheilkd 1906, 44: 428-442.

3. Harada, Y. Beitrage Zur Klinishchen Kenntnis Von Nichteitriger Choroiditis (Choroiditis Diffusa Acuta). Acta Soc Ophthalmol Jpn 1926, 30: 356-378.

4. Koyanagi, Y. Dysakusis, Alopecia Und Poliosis Bei Schwerer Uveitis Nicht Traumatischen Ursprungs. Klin Monatsbl Augenheilkd 1929, 82: 194-211.

5. Snyder, D.A. \& Tessler, H.H. Vogt-Koyanagi-Harada syndrome. Am J Ophthalmol 1980, 90: 69-75.

6. Ohno, S., Char, D.H., Kimura, S.J. \& O'Connor, G.R. HLA in Vogt-Koyanagi-Harada syndrome. $N$ Engl $J$ Med 1976: 295-788. the VKH syndrome which showed cytotoxic activity against P-36 human melanoma cells. ${ }^{10}$ Autoimmune processes may alter the cell surface components of melanocytes in such patients, so that they share a cross reacting component with melanoma cells, possibly leading to their destruction by T-lymphocytes. It is of interest to note the development of diabetes mellitus in our patient about four years before, while hypothyroidism was noted during the first admission to this hospital. Whilst the association could be entirely fortuitous, viral infections have been suggested as a possible precipitating factor in insulin dependent diabetes mellitus (IDDM) with activated T-lymphocytes found in the pancreatic islets of newly diagnosed patients with IDDM. ${ }^{11}$ One can speculate that a microbial agent or antigen which alters the cell surface component in the skin and uveal tract may also alter the beta cells in the pancreas and follicular epithelial cells in the thyroid gland leading to endocrine disturbance in this condition. The contribution of these endocrinopathies to the ophthalmic, dermal and neurological manifestations of VKH syndrome in our patient is conjectural. The association with paraparesis, raised CSF protein, antiganglioside and anti-myelin antibodies in the serum ${ }^{8}$ were previously reported in VKH syndrome. Even though it is conjectural, autoimmune mechanisms may have contributed to the neural tissue damage in this patient.

In view of the reported abnormal immunological functions and the predictable involvement of multiple seemingly diverse tissues, it is suggested that further patients with the VKH syndrome should be studied for mechanisms demonstrating autoimmunity, and for possible associations with autoimmune disorders.

7. Iverson, T.H. \& Sverrisson, T. Vogt-Koyanagi-Harada syndrome - a case report. Acta Ophthalmol 1986, 64: 235-238.

8. Manor, R.S., Livini, E. \& Cohen, S. Cell-mediated immunity to human myelin basic protein in VogtKoyanagi-Harada syndrome. Invest Ophthalmol Vis Sci 1979, 18: 204-206.

9. Moschellav, S., Pillsbury, D.M. \& Hurly, H.J. (eds) Dermatology, vol. II. W.B. Saunders Company, Philadelphia, 1975, p. 1120.

10. Maezewa, N., Yano, A., Taniguchi, M. \& Kojima, S. Role of cytotoxic T-lymphocytes in the pathogenesis of Vogt-Koyanagi-Harada disease. Ophthalmologica 1982, 185: $179-186$.

11. Hitchcock, C.L., Riley, W.J., Alamo, A., Pyka, R. \& Maclaren, N.K. Lymphocyte subsets and activation in prediabetes. Diabetes 1986, 35: 1416-1422. 